\title{
Xenogeneic support for the recovery of human donor organs
}

John D. O'Neill, PhD, ${ }^{\mathrm{a}}$ Brandon A. Guenthart, MD, ${ }^{\mathrm{b}}$ Ahmed E. Hozain, MD, ${ }^{\mathrm{c}}$ and Matthew Bacchetta, MD, MBA, MA ${ }^{\mathrm{d}, \mathrm{e}, \mathrm{f}}$

Feature Editor's Introduction-In this Invited Expert Opinion article, Bacchetta and colleagues provide an excellent overview of a novel method of cross-circulation ex-vivo lung perfusion (EVLP) using xenographic support. Although there are significant barriers that need to be overcome, the authors provide a good review of the ethical considerations, biosafety and immunological concerns, and logistical issues that must be addressed before this technique could be adopted in clinical practice. This is a potentially transformative approach that could have significant clinical impact in enhancing the recovery of donor organs and increasing the number of donor lungs available for transplant. Intact swine liver and kidney function as well as circulating cytokines, growth factors, and reparative signals are potential advantages over isolated EVLP. The authors are experts in lung transplantation and cross-circulation ex vivo lung perfusion using xenographic support. The authors have been able to prevent hyperacute rejection during xenographic cross-circulation by depleting complement activity using recombinant cobra venom factor. This novel technique will be of interest to readers. Potential areas of discussion include the ethical considerations concerning gene editing in transplantation as well as concerns about zoonotic disease transmission in light of the current coronavirus disease 2019 (COVID-19) pandemic and concerns about a zoonotic source. While lung transplantation continues to evolve with improvements in surgical techniques and organ preservation, there continues to be a critical shortage of available donor lungs, and a substantial number continue to be discarded due to questionable quality. The authors have shown that donor lungs can improve after xenographic crosscirculation, despite being declined for transplant on

\footnotetext{
From the Departments of ${ }^{\mathrm{a} C e l l}$ Biology and ${ }^{\mathrm{c}}$ Surgery, State University of New York Downstate Medical Center, SUNY Downstate Health Sciences University, Brook-

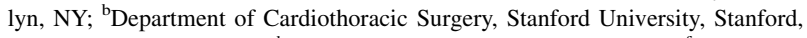
Calif; and Departments of ${ }^{\mathrm{d}}$ Thoracic Surgery, ${ }^{\mathrm{e}}$ Cardiac Surgery, and ${ }^{\mathrm{f}}$ Biomedical Engineering, Vanderbilt University, Nashville, Tenn.

Received for publication March 9, 2021; revisions received June 16, 2021; accepted for publication July 9, 2021; available ahead of print Sept 6, 2021.

Address for reprints: Matthew Bacchetta, MD, MBA, MA, Department of Thoracic Surgery, 609 Oxford House, 1313 21st Ave South, Nashville, TN 37232 (E-mail: matthew.bacchetta@vumc.org).

J Thorac Cardiovasc Surg 2022;163:1563-70

0022-5223/\$36.00

Copyright (c) 2021 by The American Association for Thoracic Surgery

https://doi.org/10.1016/j.jtcvs.2021.07.055
}

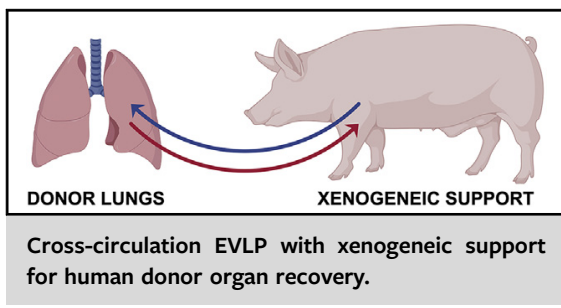

CENTRAL MESSAGE

Advanced EVLP with xenogeneic

support could complement ma-

chine perfusion modalities such

as isolated EVLP to expand

donor organ recovery and in-

crease the number of lungs

available for transplantation.

This Invited Expert Opinion provides a perspective on the following paper Source article: Nature. 2020 Jul;583(7817):495 d41586-020-02080-3.

See Commentary on page 1571.

isolated EVLP, demonstrating the potential advantage of this xenographic cross-circulation technique.

\section{Jules Lin, MD}

The opportunity for patients with end-stage lung disease to receive a life-saving transplant remains highly constrained by the shortage of donor lungs. Strategies to increase the number of available organs include the use of extendedcriteria donors, generation of bioengineered lung grafts, development of genetically modified swine lung xenografts, and extracorporeal recovery of donor lungs using isolated or cross-circulation ex vivo lung perfusion (EVLP). ${ }^{1-5}$ Isolated EVLP (ie, machine perfusion) has proved the feasibility of reconditioning donor organs and enabled transplantation of hundreds of marginal lungs. Nevertheless, despite all efforts, the number of lung transplants has only modestly 
increased. ${ }^{6}$ We envision that donor organ recovery could be significantly expanded by using "xenogeneic support" (eg, cross-circulation with living swine) to repair and recover human lungs that are not being used for transplantation due to severe but reversible injuries. Here, we trace the development of advanced EVLP with xenogeneic support (Figure 1, Video Abstract) to increase the number of available donor lungs and discuss current challenges and future perspectives in donor organ recovery.

\section{NEW APPLICATION OF AN OLD CONCEPT}

In 1954, Dr C. Walton Lillehei pioneered a technique called cross-circulation, in which blood was continuously exchanged between 2 people to enable surgical repair of cardiac defects. ${ }^{7}$ During cross-circulation, the patient received biological heart-lung support from a healthy adult (often a parent of the child undergoing cardiac surgery). More than 60 years later, our group applied this concept to support an extracorporeal organ. In a proof-offeasibility study, we showed that allogeneic crosscirculation EVLP between ex vivo lungs and a swine provided robust systemic physiologic support to an extracorporeal organ. ${ }^{8}$ In a subsequent study, we extended the durability of EVLP from hours to days by showing that allogeneic cross-circulation EVLP with a swine can support lungs ex vivo for 4 days. ${ }^{9}$ All physiological parameters assessed, including gas exchange capacity (arterial oxygen tension/inspired oxygen fraction), compliance, $\mathrm{pH}$, and lactate, were maintained within normal ranges. Lung injury scoring based on histopathologic evaluations of edema,

\section{XENOGENEIC SUPPORT FOR DONOR ORGAN RECOVERY Advanced cross-circulation EVLP}

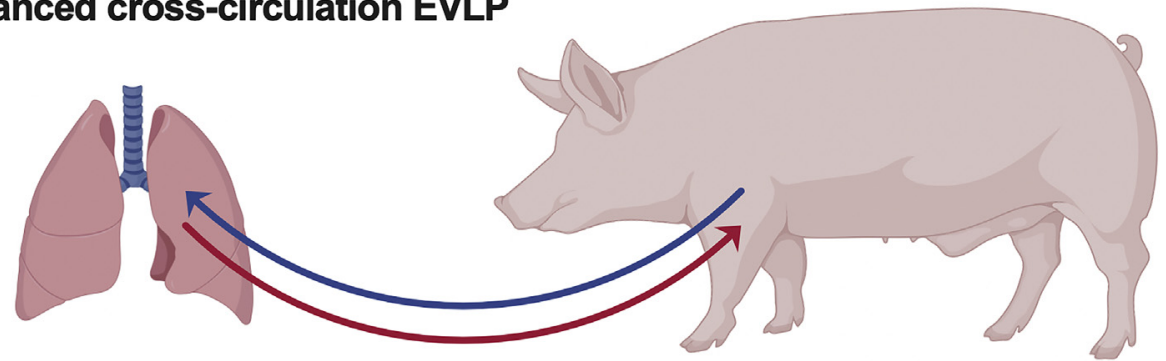

\section{FEATURES}<smiles>C1CCCCC1</smiles>

\section{Immune modulation}

Maladaptive immune responses prevented by standard immune suppression drugs and complement depletion

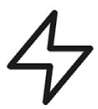

\section{Energy substrates}

Nutrients provided by glucoregulation and carbohydrate metabolism of swine liver and pancreas

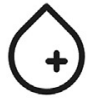

Circulating repair factors

Systemic supply of cytokines, growth factors, hormones, exosomes, and other reparative signals

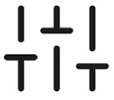

Biochemical regulation

Homeostatic balance of physiologic variables: acid-base, ions, fluid balance, neuroendocrine axes

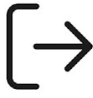

\section{Metabolic} clearance

Elimination of lactate, urea, other metabol-

ites and toxins by swine liver and kidneys

\section{BENEFITS}

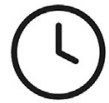

Increased recovery time

Continuous stable normothermic ex vivo lung perfusion for up to 4 days, possibly longer

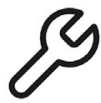

Enabled interventions

Therapeutic treatment and functional evaluations can be performed throughout organ recovery

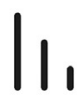

Reduced inflammation

Decreased interferons, interleukins, tumor necrosis factors

(except IL-6 in BAL fluid, IFNY in serum)

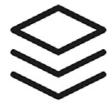

Reduced lung injury score

Decreased airway secretions, edema, infiltration, apoptosis; improved recruitment and histology

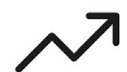

Improved function

$\uparrow$ Gas exchange

$\uparrow$ Compliance

$\downarrow$ Peak airway pressure

Restored blood-air barrier

FIGURE 1. Conceptual schematic with features and benefits of cross-circulation EVLP with xenogeneic support for the recovery of human donor organs. $E V L P$, Ex vivo lung perfusion; $I L-6$, interleukin 6; $B A L$, bronchoalveolar lavage; $I F N \gamma$, interferon gamma. 
infiltration, and apoptosis indicated minimal or no differences from healthy control lungs. Overall, these studies demonstrated the utility of systemic physiologic support provided by cross-circulation EVLP with a swine and served as the foundation for an interventional platform to investigate the recovery and regeneration of injured lungs over longer recovery times.

To assess the feasibility of using swine support to recover injured organs, we evaluated swine lungs ex vivo in 2 clinically relevant models: (1) prolonged ischemia-reperfusion injury ${ }^{8}$ and (2) gastric aspiration. ${ }^{10}$ Lungs that suffered prolonged ischemia-reperfusion injury showed functional and morphologic derangements, including consolidation, edema, inflammation, a 7-fold increase in composite injury score, and a $31 \%$ decrease in gas exchange capacity. However, after 36 hours of allogeneic cross-circulation EVLP, ex vivo lungs fully recovered: airway secretions cleared, edema resolved, and barrier function was reestablished. The gas exchange capacity, histomorphology, and composite injury score of recovered lungs were consistent with those of healthy lungs. Notably, normalization of ischemia-reperfusion injury was achieved without therapeutic intervention, but only after 24 hours of crosscirculation support, which confirmed the necessity and benefit of prolonged systemic support to restore normal function to injured lungs ex vivo.

In a subsequent study, gastric aspiration, a deleterious insult to the pulmonary epithelium that often renders lungs unusable for transplantation at the time of procurement, was induced in a reproducible swine model to assess the ability of allogeneic cross-circulation support to enable the recovery of severely damaged lungs. Gastric aspiration led to pneumonitis with dramatic inflammatory response, cellular disruption, edema, hemorrhage, a 45-fold increase in composite injury score, and a precipitous $89 \%$ decline in gas exchange capacity. To facilitate recovery, a regimen of therapeutic interventions comprising bronchoalveolar lavage (BAL), surfactant replacement, and alveolar recruitment was performed at 2, 4, 6, 12, and 18 hours of crosscirculation EVLP. After 36 hours, lungs recovered 83\% gas exchange capacity and showed evidence of cellular regeneration in the airway and vascular compartments. In BAL fluid, concentrations of interferon- $\gamma$, interleukin (IL)-1 $\beta$, IL-6, IL-8, tumor necrosis factor- $\alpha$, and other inflammatory cytokines decreased. Functional components of the alveolar-capillary membrane such as aquaporin 5 (water channel protein), ZO-1 (microvascular tight junction protein), and ZO-3 (epithelial tight junction protein), which were drastically reduced after gastric aspiration, were restored to expression patterns resembling those in control lungs. Altogether, cross-circulation EVLP enabled significant cellular regeneration and functional restoration of the blood-air barrier that correlated with improved respiratory performance of ex vivo lungs. Furthermore, as previous attempts to recover lungs injured by gastric aspiration using isolated EVLP have been unsuccessful, ${ }^{11}$ gastric aspiration remains a contraindication for standard EVLP practice. ${ }^{12}$ Our studies confirmed that allogeneic cross-circulation EVLP can regenerate severely injured lungs and thus could potentially be used to expand ex vivo donor organ recovery.

\section{Xenogeneic Support for Ex Vivo Human Organ Recovery}

Informed by a series of studies demonstrating clear benefit of allogeneic cross-circulation EVLP for the recovery of injured lungs ex vivo in a large animal model, we investigated the translational potential of crosscirculation EVLP to recover human lungs declined for transplantation. ${ }^{5}$ We hypothesized that cross-circulation EVLP with xenogeneic support (wild-type Yorkshire swine, mean age: 6 months, mean weight: $62 \mathrm{~kg}$ ) could be used to recover injured human lungs deemed unsuitable for immediate transplantation (Figure 2, A). In this study, human lungs that were declined for transplantation due to pulmonary contusions, aspiration pneumonitis, or multilobar consolidation were procured and sustained an average cold ischemia time of 20 hours before initiation of advanced EVLP with xenogeneic support (ie, ischemia times far exceeding those accepted in current clinical practice $^{12}$ ). To limit maladaptive immune responses, standard immunosuppressive drugs (mycophenolate, methylprednisolone, tacrolimus) were used in combination with a nontoxic complement depletion protein (recombinant cobra venom factor). Hyperacute rejection, a process mediated by complement activation in the allograft, was prevented by administration of recombinant cobra venom factor. Before xenogeneic cross-circulation, complement depletion was confirmed by complement total (CH50) assay.

After 24 hours of cross-circulation EVLP with xenogeneic support, average gas exchange capacity increased by $117 \%$, compliance increased by $185 \%$, and peak inspiratory pressure decreased by $16 \%$, with negligible changes in lung weight, $\mathrm{pH}$, and lactate. Consolidation resolved, aeration improved, and mean composite injury score decreased by $30 \%$ (Figure 2, B). Inflammatory cytokines in BAL fluid and serum also decreased, except IL-6 in BAL fluid and interferon- $\gamma$ in serum, which increased. No complement protein deposition, large-vessel thrombosis, thrombotic microangiopathy, or other indication of hyperacute rejection was observed in postoperative histology or immunohistochemical staining. (In control studies without immunosuppression and complement depletion, hyperacute rejection characterized by diffuse thrombosis and graft failure occurred within 1 hour of xenogeneic cross-circulation.) Importantly, endogenous regenerative lung stem cell populations, including $\mathrm{p} 63^{+}$basal cells and alpha smooth muscle actin $^{+}$submucosal gland myoepithelial cells, were retained, 
A
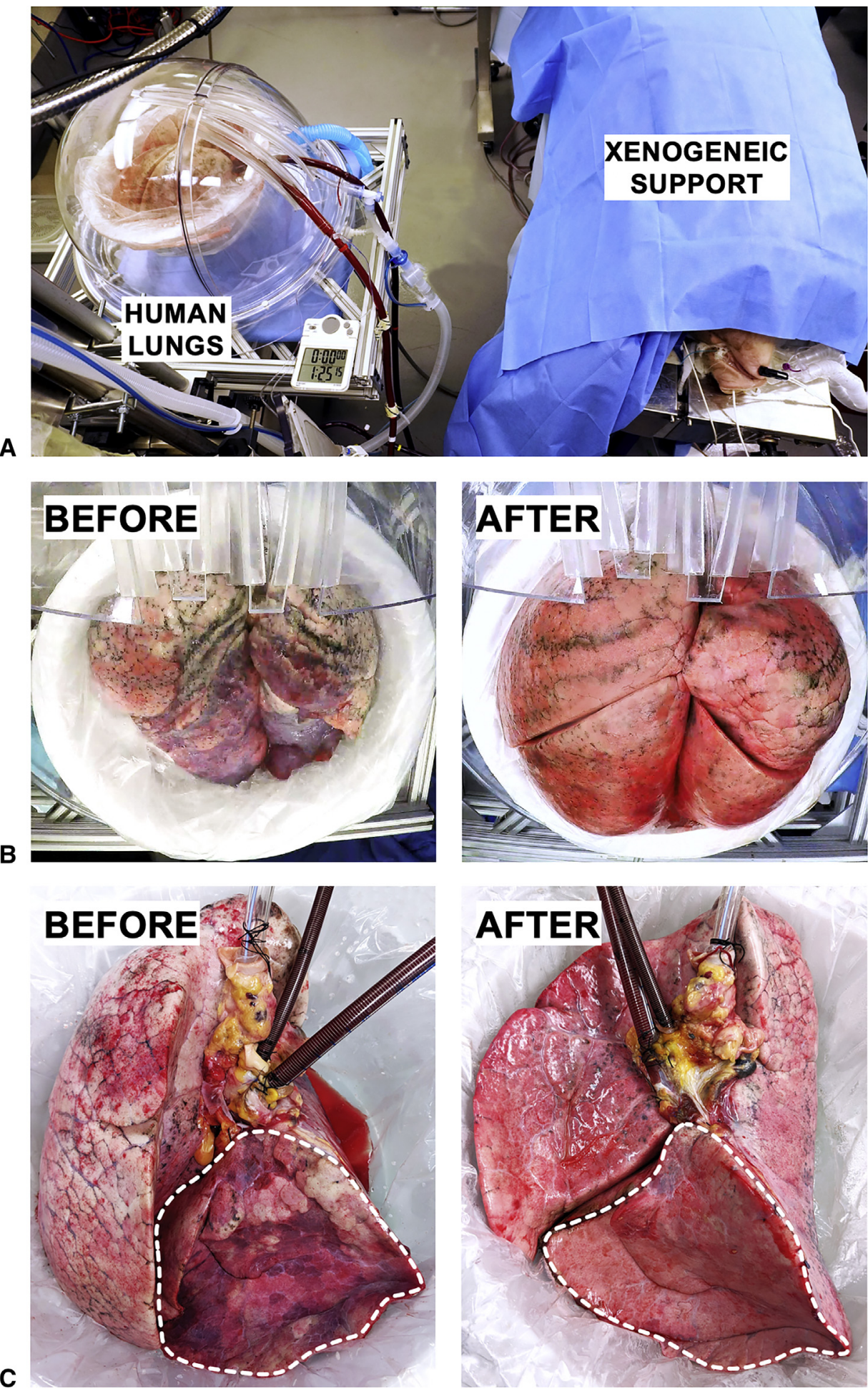

FIGURE 2. Setup and outcomes of cross-circulation EVLP with xenogeneic support. A, Photograph of experimental setup during recovery of human lungs. B, Photographs of human double-lung block that was declined for transplantation and offered for research: before ( 0 hours) and after (24 hours) recovery with xenogeneic support. C, Photographs of human single lung that failed to recover on conventional clinical EVLP and was offered for research: before (0 hours) and after (24 hours) recovery with xenogeneic support. Dashed lines highlight successful recruitment and recovery of the lower lobe. EVLP, Ex vivo lung perfusion. 
suggesting that xenogeneic support preserves intrinsic cellular repair mechanisms needed to regenerate injured lungs.

In one exemplary case, a lung that failed to recover on 5 hours of clinical EVLP (isolated machine perfusion) was declined for transplantation by multiple centers, offered for research, and transported to our laboratory. Before the initiation of xenogeneic cross-circulation, this lung sustained 27.5 hours of explant time (ie, 5 hours of clinical EVLP plus 2 cold ischemia times totaling 22.5 hours). Remarkably, after 24 hours of xenogeneic support, significant functional and structural recovery was achieved: consolidation resolved, oxygenation increased by $170 \%$, compliance increased by $21 \%$, and composite injury score decreased by $56 \%$ (Figure $2, C$ ). Such drastic improvement of a lung not otherwise salvageable by current state-of-the-art EVLP is a powerful testament to the benefits of xenogeneic support. Although a number of safety, technical, and logistical challenges remain to be addressed before clinical translation, this study highlights the great potential for cross-circulation EVLP with xenogeneic support to complement isolated EVLP modalities and effectively expand ex vivo organ recovery.

\section{FUTURE PERSPECTIVES}

The use of cross-circulation EVLP with xenogeneic support to repair human donor lungs is a disruptive paradigm shift in organ recovery that integrates advancements in ex vivo perfusion, genetic engineering, and transplant immunology to overcome barriers to resolving the organ shortage crisis. Our previous studies have shown that cross-circulation EVLP with xenogeneic support can overcome the intrinsic limitations of isolated machine perfusion by providing systemic physiologic regulation to ex vivo donor organs during recovery. Furthermore, efforts to enhance isolated EVLP, eg, with dialysis, have shown limited ability to improve graft function. ${ }^{13}$ The cellular repair and regeneration processes underlying functional recovery involve complex physiological interactions, including intrinsic communication between different organs. Such "organ crosstalk," which is mediated by mechanical, soluble, and cellular mechanisms,${ }^{14}$ and essential for homeostasis, is challenging to recreate artificially but enabled by swine systemic support via crosscirculation. Emerging clinical and experimental evidence suggest that crosstalk between lungs and other organs can facilitate cellular adaptive responses and repair processes through an array of interactions, including hormones (eg, kidney-lung crosstalk through the renin-angiotensin-aldosterone system), cytokines (eg, IL-10), and other signals (eg, peptides, small molecules, growth factors, adipokines, lipid mediators). ${ }^{15,16}$ Circulating extracellular vesicles (eg, exosomes, apoptotic bodies) have been shown to transport microRNAs that mediate inflammation, apoptosis, proliferation, and mobilization of bone marrow and other progenitor cells and induce systemic response to injury that supports cell and tissue repair. ${ }^{17,18}$ During xenogeneic support, exposure to swine antigens is transient, unlike the chronic xenoantigen exposure and related complications that continue to present major challenges for xenotransplantation. As the field continues to work toward the goal of delivering lung xenografts that are safe for transplantation into humans, we envision that advanced EVLP with xenogeneic support could be used to recover significant numbers of donor lungs that: (1) do not meet conventional isolated EVLP criteria, or (2) fail to recover on isolated EVLP (Figure 3). Herein, we discuss key considerations for the clinical translation of organ recovery systems using xenogeneic support.

\section{Ethical Considerations}

The use of xenogeneic support to expand the pool of donor lungs and offer transplantation to more patients involves important ethical considerations concerning animal use, patients, and public health. Due to the potential lifesaving benefits for patients whose needs cannot be otherwise met, the development of genetically modified swine to supply organs for xenotransplantation has been ethically justified. ${ }^{19}$ Key ethical considerations for swine genome editing have been extensively reviewed ${ }^{20-22}$ and include human health, risks and uncertainty, animal welfare and dignity, environmental issues, and public acceptability. Notably, in 2020, the Food and Drug Administration (FDA) approved "for both human food and potential therapeutic uses" the first intentional genomic alteration in a line of domestic pigs, ${ }^{23}$ underscoring the acceptability of swine genome editing for therapeutic use. Before the first-in-human studies, ethical standards and procedures, including informed consent and other safeguards, must be established to ensure that the lifelong dignity, rights, and welfare of patients who receive an organ recovered using xenogeneic support are protected. Importantly, patients who do not consent to receipt should not be disadvantaged. Expert advisory committees may facilitate development of ethical guidance for the design and conduct of human studies, including essential precautionary measures and requirements to initiate clinical trials.

\section{Safety Considerations}

The primary safety concerns for xenogeneic support pertain to biosafety and cross-species immunology. Advances in genetic engineering, especially CRIPSR-Cas9 gene editing, have accelerated the generation of genetically modified swine to address safety issues related to the use of swine in organ transplantation applications. Swine genome editing, including insertion and deletion of targeted genes, can improve safety by reducing immunological incompatibilities and risk of cross-species transmission of porcine 

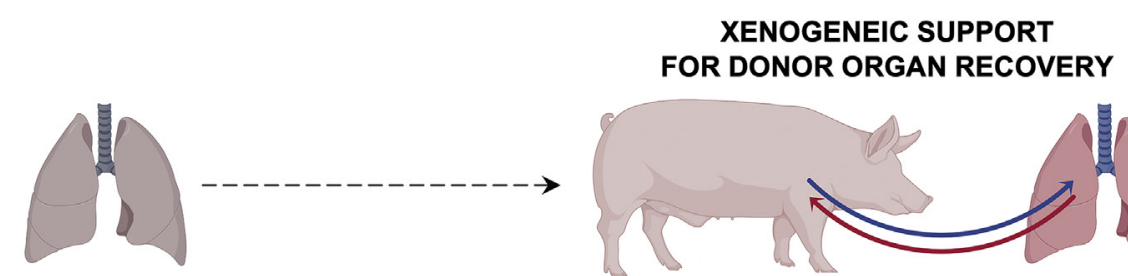

Injured human lungs

A NOT meeting EVLP criteria

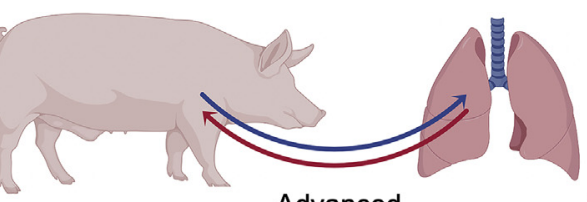

Advanced

cross-circulation EVLP

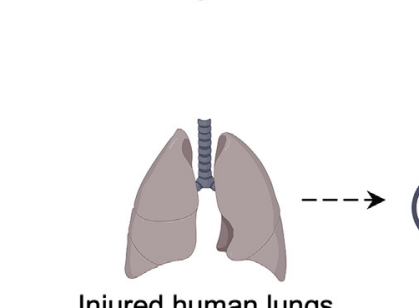

Injured human lungs

B meeting EVLP criteria
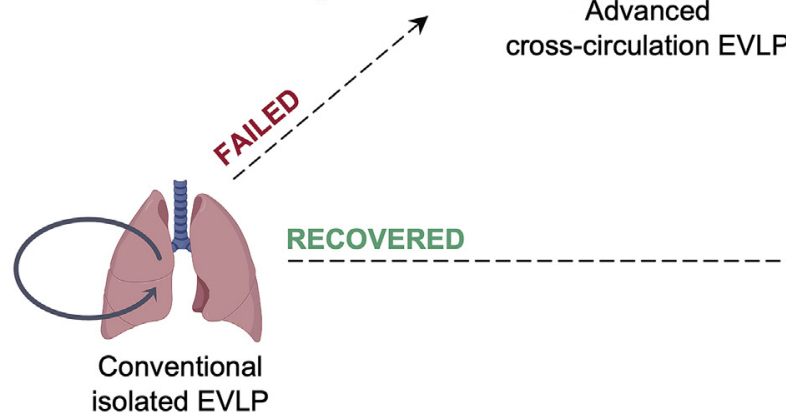

FIGURE 3. Envisioned translational application of advanced cross-circulation EVLP with xenogeneic support to increase the number of lungs available for transplantation. A, Recovery of injured human lungs that do not initially meet conventional EVLP criteria. B, Recovery of injured human lungs that meet conventional EVLP criteria but fail to improve on conventional EVLP (isolated machine perfusion). EVLP, Ex vivo lung perfusion.

endogenous retroviruses (PERVs, gamma retroviruses incorporated into the genomes of all swine strains). Although PERV transmission to humans has not been observed in vivo, infection of human cells has been reported in vitro. The successful generation of PERV-inactivated swine with all 62 copies of PERVs inactivated ${ }^{24}$ is a major step toward eliminating the biosafety risk of PERV transmission. Risk of transmission of other infectious zoonoses (eg, porcine adenovirus, porcine lymphotropic herpesvirus) ${ }^{25}$ can be mitigated by designated pathogen-free barrier facilities that screen medical-grade swine for exclusion list viruses, bacteria, and parasites using polymerase chain reaction and next-generation sequencing methods. ${ }^{26}$ Microbiologic assays and unbiased metagenomic sequencing techniques can be used to identify and exclude pathogens from breeding herds, and to monitor support swine and organ recipients for infections even if asymptomatic. Although swine genome editing has been used to mitigate risk of PERV transmission, concern for zoonotic infections cannot be discounted, and further investigation is required.

To improve immunocompatibility and reduce adverse immune reactions such as hyperacute rejection and acute humoral rejection, genetically modified swine with multiple gene knockouts (targeted gene deletions) have been generated. The presence of the xenoantigen galactosealpha-1,3-galactose (alpha Gal), which is expressed by alpha-1,3-galactosyltransferase (GalT, coded by the gene GGTA1) in pigs but not expressed in humans, is the primary cause of hyperacute rejection. Notably, successful generation of GalT knockout swine lacking the alpha Gal epitope led to approval from the FDA of GalSafe swine for potential therapeutic use, ${ }^{23}$ an important regulatory precedent for use of genetically modified swine in biomedical applications such as xenogeneic cross-circulation. While use of GalT knockout swine can significantly reduce the risk of hyperacute rejection, other swine antigens not expressed by humans, such as $\mathrm{N}$-glycolylneuraminic acid (produced by cytidine monophospho- $N$-acetylneuraminic acid hydroxylase, which is coded by the gene CMAH) and SDa glycan (produced by beta-1,4- $\mathrm{N}$-acetyl-galactosaminyltransferase 2, which is coded by the gene B4GALNT2), can lead to acute humoral rejection. Consequently, swine with multiple knockouts, such as the triple knockout of GGTA1, CMAH, and B4GALNT2, have been generated to reduce xenoreactivity. ${ }^{27}$ Insertion of human transgenes into the swine genome to generate "humanized" swine has also been achieved for a variety of human transgenes related to immune response, including CD39, CD47, thrombomodulin, tissue factor pathway inhibitor, and others. As the list of targeted genes to delete or insert is expanded and refined, safety profiles will continue to improve. Ultimately, clinical data will inform the optimal genetic modifications required for support swine, and the surveillance strategies required for transplant recipients.

The FDA defines "any procedure that involves human contact with fluids, cells, or organs that had previous contact with a non-human animal source" as xenotransplantation. ${ }^{28}$ Notably, the immunology of transient swine support for a human organ is different than that of permanent transplantation of a swine organ into a human, especially in the chronic setting, and regulatory requirements for advanced EVLP with xenogeneic support are not yet defined. Lung transplant immunology may provide important insights, as long-term outcomes of lung transplantation are negatively impacted by chronic rejection and development of 
bronchiolitis obliterans syndrome. Additional environmental and procedural safety measures will be required to ensure compliance and maintenance of sterility during xenogeneic support, wherein a live animal and ex vivo human organ intended for transplantation may be in proximity for hours to days. Immunosuppression strategies such as Tcell costimulation blockade and lymphodepletion can help further reduce detrimental immune responses, but reliance on unapproved drugs such as complement depleting cobra venom factor would introduce additional safety considerations. Since immune activity plays such a pivotal role in recovery after injury and rejection after transplant, deeper understanding of the immunologic effects of residual swine antigens, antibodies, and cells in human lungs after crosscirculation EVLP with xenogeneic support will be crucial to establish robust safety profiles.

\section{Practical Considerations}

We envision that donor organs initially declined for transplantation but meeting criteria for xenogeneic support (eg, failure to improve on conventional EVLP) could be sent to a dedicated organ recovery center specialized in advanced cross-circulation EVLP. Organ recovery centers would be equipped with "organ intensive care units" and animal care facilities and staffed by medical professionals trained in surgery, ex vivo organ perfusion, and veterinary care. Live monitoring of organs "in recovery" could provide detailed information about recovery status to lung transplant surgeons through an online portal in real time, facilitating organ assessment (visualization and functional evaluation), clinical decision-making, and logistical planning and coordination at the recipient site. Upon admission of lungs to the "organ intensive care unit," recovery protocols indicated for the relevant injury etiology would be implemented, including guidelines for lung-protective ventilation strategies and therapeutic interventions during xenogeneic support. Precision medicine principles that use molecular diagnostics, noninvasive imaging, theranostics, and/or therapeutics tailored to each organ could offer highly effective treatment options that may be "personalized" to optimize recovery of each organ. Xenogeneic support could also facilitate translational development of emerging strategies to personalize organ grafts prior to transplantation, including immunomodification, gene or cell delivery, or advanced therapies such as cell replacement ${ }^{29}$ with patient-specific stem cells.

\section{CONCLUSIONS}

Cross-circulation EVLP with xenogeneic support is a transformative approach to increase the number of lungs for transplantation by recovering donor organs that are not currently used for transplantation due to severe but reversible injuries. Future research and development driven by collaborations among physicians, surgeons, perfusionists, bioengineers, immunologists, regulatory experts, and industry partners will establish the safety and efficacy of EVLP with xenogeneic support and advance this promising technology toward clinical translation.

\section{Conflict of Interest Statement}

J.D.O., B.A.G., and M.B. are coinventors of a patent application (US20190141985A1) titled "Cross-circulation platform for recovery, regeneration, and maintenance of extracorporeal organs." A.E.H. reported no conflicts of interest.

The Journal policy requires editors and reviewers to disclose conflicts of interest and to decline handling or reviewing manuscripts for which they may have a conflict of interest. The editors and reviewers of this article have no conflicts of interest.

\section{References}

1. Nichols JE, La Francesca S, Niles JA, Vega SP, Argueta LB, Frank L, et al. Production and transplantation of bioengineered lung into a large-animal model. Sci Transl Med. 2018;10:1-12.

2. Sykes M, Sachs DH. Transplanting organs from pigs to humans. Sci Immunol. 2019;4:1-12.

3. Neizer H, Singh GB, Gupta S, Singh SK. Addressing donor-organ shortages using extended criteria in lung transplantation. Ann Cardiothorac Surg. 2020;9: 49-50.

4. Cypel M, Yeung JC, Liu M, Anraku M, Chen F, Karolak W, et al. Normothermic ex vivo lung perfusion in clinical lung transplantation. $N$ Engl J Med. 2011;364: 1431-40.

5. Hozain AE, O'Neill JD, Pinezich MR, Tipograf Y, Donocoff R, Cunningham KM, et al. Xenogeneic cross-circulation for extracorporeal recovery of injured human lungs. Nat Med. 2020;26:1102-13.

6. Valapour M, Lehr CJ, Skeans MA, Smith JM, Uccellini K, Goff R, et al. OPTN/ SRTR 2018 Annual Data Report: Lung. Scientific Registry of Transplant Recipients. Available at: http://srtr.transplant.hrsa.gov/annual_reports/Default.aspx. Accessed February 12, 2021.

7. Gott VL, Shumway NE. Cross-circulation: a milestone in cardiac surgery. $J$ Thorac Cardiovasc Surg. 2004;127:617.

8. O’Neill JD, Guenthart BA, Kim J, Chicotka S, Queen D, Fung K, et al. Cross-circulation for extracorporeal support and recovery of the lung. Nat Biomed Eng. 2017; 1:1-15.

9. Hozain AE, Tipograf Y, Pinezich MR, Cunningham KM, Donocoff R, Queen D, et al. Multi-day maintenance of extracorporeal lungs using cross-circulation with conscious swine. J Thorac Cardiovasc Surg. 2019, 159:1640-53.

10. Guenthart BA, O'Neill JD, Kim J, Queen D, Chicotka S, Fung K, et al. Regeneration of severely damaged lungs using an interventional cross-circulation platform. Nat Commun. 2019;10:1-16.

11. Khalifé-Hocquemiller T, Sage E, Dorfmuller P, Eddahibi S, Fadel E. 394 Ex vivo perfusion worsened lung injuries induced by gastric acid aspiration in pigs. $J$ Heart Lung Transplant. 2011;30:S135-6.

12. US National Library of Medicine. Increasing Lung Transplant Availability Using Normothermic Ex Vivo Lung Perfusion (EVLP) at a Dedicated EVLP Facility. Identifier NCT03641677. Available at: https://clinicaltrials.gov/ct2/show/ NCT03641677. Accessed March 1, 2021.

13. Buchko MT, Himmat S, Stewart CJ, Hatami S, Dromparis P, Adam BA, et al Continuous hemodialysis does not improve graft function during ex vivo lung perfusion over 24 hours. Transplant Proc. 2019;51:2022-8.

14. Armutcu F. Organ crosstalk: the potent roles of inflammation and fibrotic changes in the course of organ interactions. Inflamm Res. 2019;68:825-39.

15. Husain-Syed F, McCullough PA, Birk HW, Renker M, Brocca A, Seeger W, et al. Cardio-pulmonary-renal interactions. J Am Coll Cardiol. 2015;65 2433-48.

16. Priest $\mathrm{C}$, Tontonoz P. Inter-organ cross-talk in metabolic syndrome. Nat Metab. 2019;1:1177-88 
17. Zhu Z, Zhang D, Lee H, Menon AA, Wu J, Hu K, et al. Macrophage-derived apoptotic bodies promote the proliferation of the recipient cells via shuttling microRNA-221/222. J Leukoc Biol. 2017;101:1349-59.

18. Cheng M, Yang J, Zhao X, Zhang E, Zeng Q, Yang Y, et al. Circulating myocardial microRNAs from infarcted hearts are carried in exosomes and mobilise bone marrow progenitor cells. Nat Commun. 2019;10:1-9.

19. Cengiz N, Wareham CS. Ethical considerations in xenotransplantation: a review. Curr Opin Organ Transplant. 2020;25:483-8.

20. European Group on Ethics in Science and New Technologies (European Commission). Ethics of genome editing. Available at: https://ec.europa.eu/info/ research-and-innovation/strategy/support-policy-making/scientific-suppo rt-eu-policies/ege_en. Accessed June 13, 2021.

21. Ayanoğlu FB, Elçin AE, Elçin YM. Bioethical issues in genome editing by CRISPR-Cas9 technology. Turk J Biol. 2020;44:110-20.

22. de Graeff N, Jongsma KR, Johnston J, Hartley S, Bredenoord AL. The ethics of genome editing in non-human animals: a systematic review of reasons reported in the academic literature. Phil Trans R Soc B. 2019;374:20180106.

23. US Food \& Drug Administration. FDA Approves First-of-its-Kind Intentional Genomic Alteration in Line of Domestic Pigs for Both Human Food, Potential Therapeutic Uses. Available at: https://www.fda.gov/news-events/press-ann ouncements/fda-approves-first-its-kind-intentional-genomic-alteration-line-dome stic-pigs-both-human-food. Accessed February 12, 2021.

24. Niu D, Wei HJ, Lin L, George H, Wang T, Lee IH, et al. Inactivation of porcine endogenous retrovirus in pigs using CRISPR-Cas9. Science. 2017;357:1303-7.
25. Boneva R, Folks T. Xenotransplantation and risks of zoonotic infections. Ann Med. 2004;36:504-17.

26. Noordergraaf J, Schucker A, Martin M, Schuurman H, Ordway B, Cooley K, et al. Pathogen elimination and prevention within a regulated, designated pathogen free, closed pig herd for long-term breeding and production of xenotransplantation materials. Xenotransplantation. 2018;25:e12428.

27. Lu T, Yang B, Wang R, Qin C. Xenotransplantation: current status in preclinical research. Front Immunol. 2020;10:3060.

28. US Food \& Drug Administration. Xenotransplantation. Available at: https:// www.fda.gov/vaccines-blood-biologics/xenotransplantation. Accessed February $12,2021$.

29. Guenthart BA, O'Neill JD, Kim J, Fung K, Vunjak-Novakovic G, Bacchetta M. Cell replacement in human lung bioengineering. Heart Lung Transplant. 2019; 38:215-24.

Key Words: acute lung injury, aspiration pneumonitis, bioengineering, cross-circulation, ex vivo lung perfusion, ischemia-reperfusion injury, lung, organ crosstalk, organ donation, organ recovery, perfusion, regeneration, transplantation, xenogeneic, xenotransplantation 